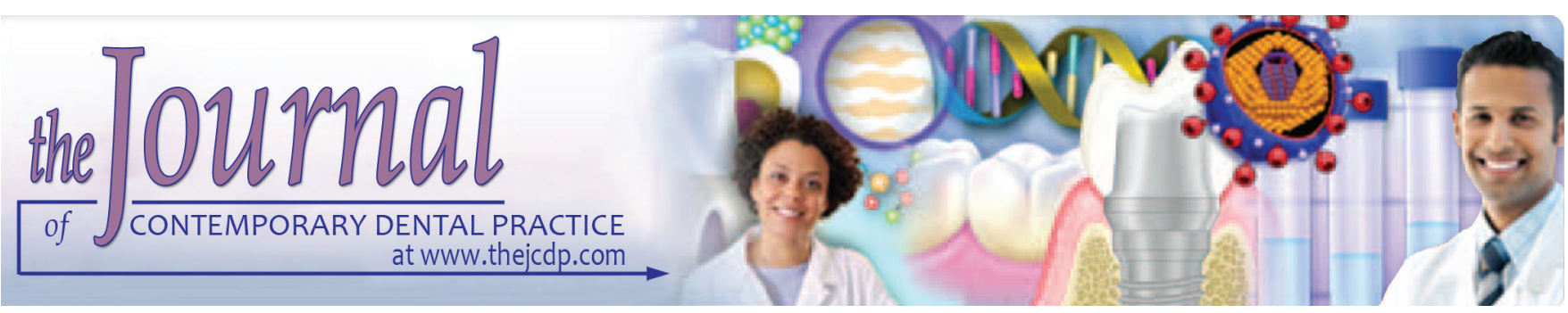

\title{
Assessment of the Effect of Orthodontic Treatment on the Periodontal Health of Endodontically Restored Tooth
}

\author{
${ }^{1}$ Md Jalaluddin, ${ }^{2}$ Vinod Goyal, ${ }^{3}$ Zuber A Naqvi, ${ }^{4}$ Bhaskar Gupta, ${ }^{5}$ Mohil M Asnani, ${ }^{6} \mathrm{Hitesh}$ M Sonigra
}

\begin{abstract}
Intorduction: Patients usually undergo orthodontic treatment for achieving ideal interocclusal relationship between the dental tissue and bony tissue along with improving the speech, mastication, and facial esthetic appearance. Literature quotes paucity in the studies evaluating the effect of orthodontic treatment on the periodontal health of endodontically treated teeth. Hence, we planned the present study to assess the effect of orthodontic treatment on the periodontal health of endodontically restored tooth.
\end{abstract}

Materials and methods: The present study included assessment of 80 patients who underwent orthodontic treatment. All the patients were divided broadly into two study groups: groups I and II. Group I included patients with the absence of endodontically treated teeth, while group II included patients which maxillary central incisors were resorted endodontically. Examination of the periodontal health of the patients was done using the community periodontal index of treatment need (CPITN) around the selected teeth. All the values were recorded during the preorthodontic time, postorthodontic time, and after the first

\footnotetext{
${ }^{1}$ Department of Periodontics and Oral Implantology, Kalinga Institute of Dental Sciences, Kalinga Institute of Industrial Technology University, Bhubaneswar, Odisha, India

${ }^{2}$ Department of Orthodontics and Dentofacial Orthopedics Institute of Dental Education \& Advance Studies, Gwalior Madhya Pradesh, India

${ }^{3}$ Department of Preventive Dental Sciences, College of Dentistry Zulfi, Majmaah University, Riyadh Province, Kingdom of Saudi Arabia

${ }^{4}$ Department of Public Health Dentistry, Hazaribag College of Dental Sciences and Hospital, Hazaribag, Jharkhand, India

${ }^{5,6}$ Department of Conservative Dentistry and Endodontics Narsinhbhai Patel Dental College \& Hospital, Visnagar, Gujarat India

Corresponding Author: Md Jalaluddin, Department of Periodontics and Oral Implantology, Kalinga Institute of Dental Sciences, Kalinga Institute of Industrial Technology University Bhubaneswar, Odisha, India, Phone: +919338131843, e-mail: jalaluddinmd@gmail.com
}

6 months of starting of the orthodontic treatment. All the results were recorded separately and analyzed.

Results: In the groups I and II, 28 and 25 patients respectively, had score of 1 , while 10 patients in group I and 12 patients in group II had score of 2 . Nonsignificant results were obtained while comparing the CPITN score in between the two study groups when measured at the pre-, intra-, and postortho time.

Conclusion: In patients undergoing orthodontic treatment, having endodontically resorted teeth, no difference exists in relation to the periodontal health.

Clinical significance: Orthodontic treatment can be safely carried in patients with endodontically restored teeth.

Keywords: Endodontically restored, Orthodontic, Periodontal.

How to cite this article: Jalaluddin M, Goyal V, Naqvi ZA, Gupta B, Asnani MM, Sonigra HM. Assessment of the Effect of Orthodontic Treatment on the Periodontal Health of Endodontically Restored Tooth. J Contemp Dent Pract 2017;18(7):587-590.

\section{Source of support: Nil \\ Conflict of interest: None}

\section{INTRODUCTION}

For the complete alignment of teeth and for achievement of ideal interocclusal relationship between the dental tissue and bony tissue, orthodontic treatment is carried out. It increases the self-confidence of the patients by improving the speech, mastication, and facial esthetic appearance of the patient. This further leads to improvement in the quality of life of the patient. ${ }^{1}$

As in the case of other treatment modalities, orthodontic treatment is associated with certain other risk factors and problems. However, if we compare these risk factors with risks involved in other surgical treatment cases, problems and their associated risks associated with orthodontic treatment are comparatively less. ${ }^{2}$ Complications and problems associated with orthodontic treatment are divided into two types: Local complication and systemic 
complication. Discoloration of teeth, demineralization of the hard tissue, periodontal pathologies include the local complications, while systemic complications include allergic reactions and infective endocarditis. ${ }^{3,4}$

For the ideal success of orthodontic treatment therapy, one of the most important contributing factors is periodontal health. The most common type of complications associated with orthodontic treatment is periodontal complications. Furthermore, good periodontal health is promoted by ideal alignment of teeth which are easier to clean and maintain. Gingivitis, periodontitis, gingival recession, or atrophy are the common periodontal complications encountered. ${ }^{5}$ Results from the past literature have shown that orthodontic forces can induce the inflammation of the periodontal tissues. ${ }^{6}$ Signs of dentin and cementum resorption are shown by teeth which have undergone endodontic therapy. Affected areas of the endodontic teeth in which resorption occurs are frequently associated with the accumulation of the granulation type of tissue. Similar type of resorption is reported to occur in the teeth which have undergone orthodontic treatment. ${ }^{4,6}$ Hence, we planned the present study to assess the effect of orthodontic treatment on the periodontal health of endodontically restored tooth.

\section{MATERIALS AND METHODS}

The present study was conducted in the Department of Orthodontics of the dental institution and included assessment of 80 patients who underwent orthodontic treatment from January 2010 to January 2016. Ethical approval was taken from Institutional Ethical Committee and written consent was obtained after explaining in detail the entire research protocol. All the patients were divided broadly into two study groups: groups I and II. Group I included patients with the absence of endodontically treated teeth while group II included patients with the presence of minimum of one endodontically restored tooth. Further, for group II patients only those patients in whom maxillary central incisors were resorted endodontically were included. Both the groups consisted of 40 patients each. Inclusion criteria for the present study included:

- Patients with complete dentition status excluding third molars

- Patients in which minimum time period from completion of orthodontic treatment to the time of evaluation is more than 1 year

- Patients with negative history of any systemic illness

- Patients without any history of known drug allergy Complete oral prophylaxis of all the patients was done before the starting of the orthodontic treatment followed by instruction for the maintenance of oral hygiene. Complete examination of the patients was done before the commencement of orthodontic treatment. Follow-up examination of the patients was done at 8-20-month time. Examination of the periodontal health of the patients was done using the community periodontal index of treatment need (CPITN) around the selected teeth using the World Health Organization probe. ${ }^{7}$ All the values were recorded during the preorthodontic time, postorthodontic time, and after the first 6 months of starting of the orthodontic treatment. Consultant and experienced periodontists were employed for the assessment of the periodontal health of all the individuals included in the study. All the results were recorded separately and analyzed by Statistical Package for the Social Sciences software version 17.0. Chi-square test and Student's t-test were used for the assessment of level of significance.

\section{RESULTS}

Preortho CPITN score of patients on both the study groups is shown in Table 1. A total of 28 patients in group I and 25 patients in group II had score of 1 , while 10 patients in group I and 12 patients in group II had score of 2 respectively. Two and three patients of groups I and II had score of 3 respectively. None of the patients of both the groups had score of 4 . Nonsignificant (NS) results were obtained while comparing the CPITN score in between the two study groups when measured at the preortho time. A total of 24 and 23 patients of groups I and II and 14 patients each of groups I and II had CPITN score of 1 and 2 respectively. None of the patients of both the study group during the orthodontic treatment had score of 4. Only two and three patients of groups I and II had score of 3 respectively (Table 2). While comparing the CPITN score in between the two study groups during the intraortho time period, NS results were obtained. A total of 22 patients each of groups I and II had CPITN score of 1 , while 15 patients of group I and 14 patients of group II had score of 2 respectively (Table 3 and Graph 1 ). Nonsignificant results were obtained while comparing the mean CPITN score in between the two study groups when measured in the postortho period.

\section{DISCUSSION}

To complete the orthodontic treatment with minimal damage to the tooth, supporting structures is one of

Table 1: Preortho CPITN score in patients of both the study groups

\begin{tabular}{llll}
\hline & \multicolumn{2}{c}{ Number of patients } & \\
\cline { 2 - 3 } CPITN score & Group I & Group II & p-value \\
\hline One & 28 & 25 & 0.25 NS \\
Two & 10 & 12 & \\
Three & 2 & 3 & \\
Four & 0 & 0 & \\
\hline Total & 40 & 40 & \\
\hline NS: & 40 & &
\end{tabular}

NS: Nonsignificant 
Table 2: Intraortho CPITN score in patients of both the study groups

\begin{tabular}{llll}
\hline & \multicolumn{2}{c}{ Number of patients } & \\
\cline { 2 - 3 } CPITN score & Group I & Group II & p-value \\
\hline One & 24 & 23 & $0.31 \mathrm{NS}$ \\
Two & 14 & 14 & \\
Three & 2 & 3 & \\
Four & 0 & 0 & \\
\hline Total & 40 & 40 & \\
\hline
\end{tabular}

NS: Nonsignificant

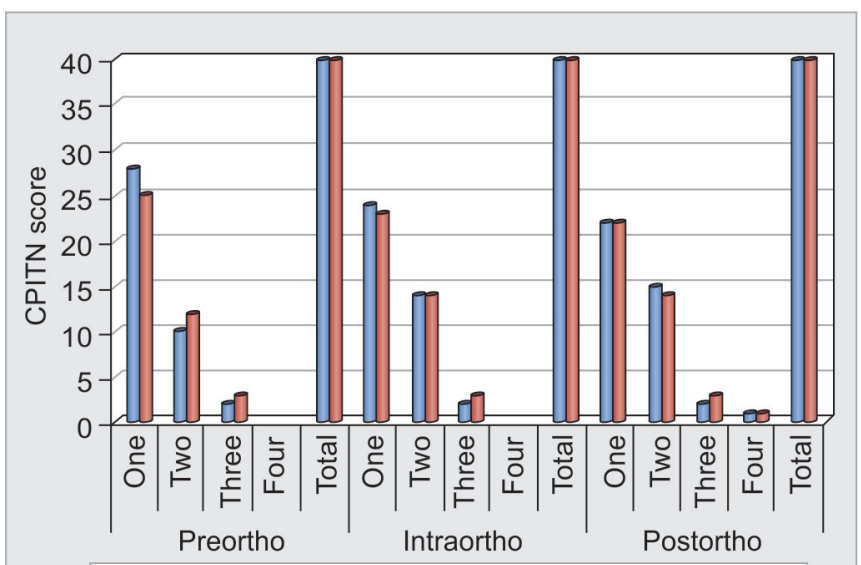

$\square$ No. of patients group A $\quad \square$ No. of patients group B

Graph 1: Pre-, intra-, and postortho CPITN score in patients of both the study groups

the primaries of the orthodontic treatment. Although unavoidable, root resorption is most commonly seen in teeth subjected to orthodontic forces. ${ }^{8}$ Variability in the individuals' biological status, genetic predisposition, and the quantity and type of orthodontic forces influences the apical root resorption. Furthermore, reported cases from the literature show that undesirable complications associated with orthodontic treatment include apical root resorption and periodontal problems. ${ }^{9}$ A treatment challenge can be imposed while performing orthodontic tooth movement on the endodontically restored teeth. Endodontically treated teeth might be present due to deep carious lesion or trauma. Rehabilitation of misaligned teeth and alignment of teeth following trauma are the possible reasons for patients seeking orthodontic treatment. Sometimes, the clinicians can be unsure regarding the precautions involved or alteration of the treatment protocol while planning the orthodontic treatment in root canal-treated teeth. ${ }^{10}$ Hence, we planned the present study to assess the effect of orthodontic treatment on the periodontal health of endodontically restored tooth.

In the present study, we observed that during the preorthodontic time period, the majority of the patients in both the study groups had a score of 1 (Table 1). During the intraortho time, the majority of patients had a score of 1 (Table 2). Furthermore, NS results were obtained while
Table 3: Postortho CPITN score in patients of both the study groups

\begin{tabular}{llll}
\hline & \multicolumn{2}{c}{ Number of patients } & \\
\cline { 2 - 3 } CPITN score & Group I & Group II & p-value \\
\hline One & 22 & 22 & 0.46 NS \\
Two & 15 & 14 & \\
Three & 2 & 3 & \\
Four & 1 & 1 & \\
\hline Total & 40 & 40 & \\
\hline
\end{tabular}

NS: Nonsignificant

comparing the mean CPITN score during the pre-, intra-, and postortho time in between the two study groups (Table 3 and Graph 1). We could not find any study in the literature that has compared the periodontal health of endodontically treated teeth and vital teeth in patients undergoing orthodontic treatment. However, when compared between endodontically treated teeth and vital teeth in patients undergoing orthodontic treatment, Nasir et al ${ }^{7}$ could not find any significant difference in the degree of root resorption in between the two study groups.

Esteves et $\mathrm{al}^{11}$ radiographically assessed the degree of root resorption in patients with teeth treated with root canal therapy and in patients with untreated teeth undergoing orthodontic treatment. They assessed records of 25,000 patients, out of which, they selected 16 patients that met the inclusion and exclusion criteria. Only those cases in whom maxillary central incisor was present were selected, which was endodontically restored before the commencement of orthodontic treatment. They did not observe any significant difference between the amounts of apical root resorption in the individuals of the two study groups. Ioannidou-Marathiotou et $\mathrm{al}^{12}$ conducted a meta-analysis to assess the impact of orthodontic treatment on the resorption of endodontically restored teeth in comparison with vital teeth. They conducted a systemic review involving 18 electronic databases and searched relevant data and observed that only 6 papers met the inclusion and inclusion criteria. Less amount of root resorption was observed in the endodontically treated teeth in comparison with the vital teeth in patients undergoing orthodontic treatment.

Spurrier et $\mathrm{al}^{13}$ assessed the difference in the amount of root resorption occurring in endodontically treated teeth and vital teeth in patients undergoing orthodontic treatment. They examined a total of 43 patients and observed a significantly higher degree of root resorption in vital teeth in comparison with the teeth restored endodontically. They also observed a significant difference in the degree of resorption in control teeth in males in comparison with females. Han ${ }^{14}$ assessed the impact of orthodontic treatment on the periodontal health of patients and also comparatively evaluated the orthodontic treatment with fixed appliances (FA) with clear aligner treatment (CAT) in 
periodontitis patients. They evaluated a total of 35 patients who underwent orthodontic treatment and later underwent FA or CAT. They compared the clinical parameters at baseline and after the commencement and finishing of orthodontic treatment. They observed that after the orthodontic treatment, there was a significant improvement in the overall plaque index score, gingival index score, and the probing depth. An overall improvement in bone level was also seen. However, they observed NS difference in the bone level changes in the FA group and the CAT group. From the results, they concluded that periodontal health of the patients can be improved greatly by combined periodontal therapy and orthodontic treatment.

Nasir et $\mathrm{al}^{7}$ evaluated the impact of orthodontic treatment on the periodontal health of young patients. They assessed a total of 50 patients that were randomly selected from the department of orthodontics that were undergoing orthodontic treatment. They evaluated the CPITN of the patients to assess their periodontal health. They observed signs of periodontal diseases in patients undergoing orthodontic treatment. They observed a strong relationship between the duration of orthodontic treatment and the progression of periodontal diseases.

\section{CONCLUSION}

From the above results, the authors concluded that in the patients undergoing orthodontic treatment, having endodontically resorted teeth, no difference exists in relation to the periodontal health. However, future studies are required for further exploring this field of dentistry.

\section{REFERENCES}

1. Preoteasa, CT.; Lonescu, E.; Preoteasa, E. Risks and complications associated with orthodontic treatment. In: Bourzgui F, editor. Orthodontics - basic aspects and clinical considerations. Rijeka: Intech; 2012. p. 423-425.
2. Talic NF. Adverse effects of orthodontic treatment: a clinical perspective. Saudi Dent J 2011 Apr;23(2):55-59.

3. Sonwane S, Ganesh P, Kumar BS. Is orthodontic treatment causes bacterial endocarditis? A review based random study. Int J Mol Med Sci 2013 Jun;3(2):6-12.

4. Tripuwabhrut P, Brudvik P, Fristad I, Rethnam S. Experimental orthodontic tooth movement and extensive root resorption: periodontal and pulpal changes. Eur J Oral Sci 2010 Dec;118(6):596-603.

5. Dannan A. An update on periodontic-orthodontic interrelationships. J Indian Soc Periodontol 2010 Jul;14(1):66-71.

6. Romero M, Albi M, Bravo LA. Surgical solutions to periodontal complications of orthodontic therapy. J Clin Pediatr Dent 2000 Spring;24(3):159-163.

7. Nasir N, Ali S, Bashir U, Ullah A. Effect of orthodontic treatment of periodontal health. Pak Oral Dent J 2011 Jun;31(1): 111-114.

8. Kitada K, de Toledo A, Oho T. Increase in detachable opportunistic bacteria in oral cavity of orthodontic patients. Int J Dent Hyg 2009 May;7(2):121-125.

9. Alexander SA. Effects of orthodontic attachments on the gingival health of permanent second molars. Am J Orthod Dentofacial Orthop 1991 Oct;100(4):337-340.

10. de Souza RS, Gandini LG Jr, de Souza V, Holland R, Dezan E Jr. Influence of orthodontic dental movement on the healing process of teeth with periapical lesions. J Endod 2006 Feb;32(2):115-119.

11. Esteves T, Ramos AL, Pereira CM, Hidalgo MM. Orthodontic root resorption of endodontically treated teeth. J Endod 2007 Feb;33(2):119-122.

12. Ioannidou-Marathiotou I, Zafeiriadis AA, Papadopoulos MA. Root resorption of endodontically treated teeth following orthodontic treatment: a meta-analysis. Clin Oral Investig 2013 Oct;17(7):1733-1744.

13. Spurrier SW, Hall SH, Joondeph DR, Shapiro PA, Riedel RA. A comparison of apical root resorption during orthodontic treatment in endodontically treated and vital teeth. Am J Orthod Dentofacial Orthop 1990 Feb;97(2):130-134.

14. Han JY. A comparative study of combined periodontal and orthodontic treatment with fixed appliances and clear aligners in patients with periodontitis. J Periodont Implant Sci 2015 Dec;45(6):193-204. 